\title{
Tangence
}

\section{Autobiographie et métalangage}

\section{Patrick Imbert}

Numéro 45, octobre 1994

Authenticité et littérature personnelle

URI : https://id.erudit.org/iderudit/025822ar

DOI : https://doi.org/10.7202/025822ar

Aller au sommaire du numéro

Éditeur(s)

Tangence

ISSN

0226-9554 (imprimé)

1710-0305 (numérique)

Découvrir la revue

Citer cet article

Imbert, P. (1994). Autobiographie et métalangage. Tangence, (45), 16-25.

https://doi.org/10.7202/025822ar d'utilisation que vous pouvez consulter en ligne.

https://apropos.erudit.org/fr/usagers/politique-dutilisation/ 


\section{Autobiographie et métalangage Patrick Imbert}

Dans la mesure où tout est énonciation ou plutôt énonciation énoncée et que tout énoncé est d'une manière ou d'une autre marqué subjectivement [...] les présentes réflexions portent sur un objet qui semble échapper à toute prise de l'intelligible.

Julie Leblanc, "Action ou interaction: l'énonciation littéraire", $R S / S I$, vol. XII, n 3,1992 , p. 82

Dans cette épigraphe, Julie Leblanc souligne l'utilité et le danger de l'application de paradigmes explicatifs totalisants chers aux formalismes et à tout métalangage. Michel de Certeau ${ }^{1}$ rappelle qu'un texte historique (et qu'est-ce qu'une biographie, sinon un type de texte historique doublé parfois de roman d'apprentissage?) "substitue la représentation d'un passé à l'élucidation de l'opération institutionnelle qui le fabrique". C'est dire que le texte masque sa praxis en tant que discours sous un semblant de réel. Il en est de même d'un métalangage qui serait utilisé pour se pencher sur le genre biographique. En effet, on peut dire de ce genre qu'il tente, par le biais de l'énonciation et de l'unique, de s'arracher aux pesanteurs politico-historico-discursives consensuelles et métalinguistiques.

Ainsi, l'accord au sujet d'un certain type de métalangage permet par la représentation donnée, celle de la littérature ou de la fictionnalité en particulier, de masquer les pratiques instituantes déployant leurs rôles pour présenter les maîtres des discours et des canons. Pierre Bourdieu dans Homo Academicus ${ }^{2}$, ouvrage révélateur et rarement cité, soulève quelque peu ce voile professionnel et discursif. Parler, dans les années 1980 et 1990, de

1 Michel de Certeau, Histoire et psychanalyse: entre science et fiction, Paris, Gallimard, coll. "Folio", 1987, p. 73.

2 Pierre Bourdieu, Homo Academicus, Paris, Minuit, 1984. 
l'autobiographie, du journal intime, n'est pas plus innocent que de parler des structures formelles dans les années soixante, des horizons d'attente ou du lecteur dans les années soixante-dix ou quatre-vingt. C'est ce que souligne, parfois maladroitement, Dinesh D'Souza dans Illiberal Education ${ }^{3}$.

C'est pourquoi nous avons décidé de prendre des autobiographies qui, lorsqu'elles furent produites, étaient déja déviantes par rapport au canon défini par Philippe Lejeune ${ }^{4}$. On pense à certains faits formels comme l'utilisation du JE ou du NOM du personnage qui possède un pouvoir d'individualisation menant à un effet de réel certain. Mais il s'agit d'EFFETS de réel car rien, discursivement, ne permet d'établir l'authenticité de l'autobiographie par rapport à une version fictionnelle, sinon le fait que "le contenu énoncé soit vérifiable ou falsifiable " comme l'affirme Julie Leblanc ${ }^{5}$. Pour cela, on voit que ces vérifications, dans les cadres généraux du mensonge et de la désinformation ${ }^{6}$, ne sont pas toujours évidentes puisque récits historiques comme autobiographiques sont non seulement marqués par les découvertes freudiennes détruisant une certaine conception psychologique de l'individu (responsabilité, autonomie du savoir, pleine liberté) mais surtout par les enjeux micro- ou macrostratégiques professionnels, politiques, médiatiques.

L'autobiographie perçue dans une optique freudienne et/ou stratégico-politique représente un discours fictionnel, parfois fortement médiatisé, affirmant cependant les vertus de l'authenticité pour les tenants des langages publics ${ }^{7}$ mais s'offrant comme autre aux incursions des langages spécialisés divers: psychanalyse, déconstruction, sémiotiques. Les spécialistes construisent l'autobiographie autrement que le grand public quoiqu'ils soient eux aussi clivés et aient été formés par les écoles, les médias, etc., aux a priori des langages publics.

3 Dinesh D'Souza, Illiberal Education, New York, The Free Press, 1991.

4 Philippe Lejeune, Le pacte autobiograpbique, Paris, Seuil, 1975; Moi aussi, Paris, Seuil, 1986.

5 Julie Leblanc, "Le statut du référent dans les récits autobiographiques fictionnels et factuels", Revue canadienne de littérature comparée, vol. XIX, $\mathrm{n}^{\circ} 4$, décembre 1992, p. 523-538.

6 Patrick Imbert, "Peace and War: Public Languages, Specialized Languages and the Media ", Semiotica, vol. XCIX, $\mathrm{n}^{\text {os }} 1-2$, mars 1994, p. $29-51$.

7 On reprend les distinctions de Basil Bernstein, Class, Codes and Control, Londres, Routledge and Kegan Paul, 1971, 3 vol. 
18

Dès lors, des auteurs comme Claude Mathieu ou Marcel Godin, universitaires ou intellectuels maniant des paradigmes différents de ceux à l'œuvre dans les langages publics vont se jouer du canon. Leurs autobiographies-fictions intègrent un savoir opacifié par l'écriture fictionnelle sur le genre autobiographique même. Évidemment, ce canon autobiographique comme genre se situe dans une optique épistémologique et dans une certaine conception du signe et du rapport du signe à un extérieur. C'est cette conception qu'il faut préciser.

\section{Signe et extérieur}

What we know is not the world but stories about the world.

Stanley Fish, Is There a Text in This Class?, p. 243

Toute la révélation divine est contenue dans l'Écriture, affirme la tradition. Mais, en même temps, elle maintient que cette révélation ne peut être saisie que par l'exégèse qui donnera sa mesure à la littéralité dans son excès même. Cette exégèse est maîtrisée par le canon et ses exigences. Le Livre originel se transmet alors "sans modification" dans sa lettre, nonobstant les traductions dont Claude Tresmontant ${ }^{8}$ a montré certains errements. Pourtant le Livre pourrait exploser de significations multiples issues du mystère de cette totalité. Le secret est bien dans cette totalité de la lettre qui est l'enjeu de la lecture. Celle-ci contrôle le jeu de cette lettre originaire cachant/révélant, à répéter, mais ouverte. Ce jeu est paradoxal au départ et bientôt dualiste car il est réglé et canonisé par une institution, celle de l'Église travaillant le littéral et le spirituel.

L'excès du secret se trouve pris en charge par les règles explicitées par Agostino de Dacie au xiII siècle dans le Rotulus pugillaris (vers 1260). Il s'agit d'un canon rigide qui assied pour des siècles une pratique de lecture: sens de la lettre enseignant I'histoire, sens de l'allégorie désignant les objets de la foi, sens de la tropologie guidant l'action du point de vue moral, sens de

8 Claude Tresmontant, Le Christ hébreu, Paris, CEil, 1983. 
l'anagogie ouvrant sur les fins dernières dans le cadre de l'espérance. Ce fonctionnement est vulgarisé, notamment dans la Gesta Romanorum ${ }^{9}$ proposant des textes courts agrémentés d'un commentaire qui en désigne les significations et qui établit les liens entre le nouveau texte profane et le texte originel sacré: le valet est l'ange Gabriel, la bergère est Marie, etc.

Ce double sens lettre /esprit, dont le second terme est valorisé dans le christianisme puis par la culture occidentale laïcisée reposant toujours sur plusieurs de ses a priori, est lié au double auteur/lecteur et à une volonté d'arrêter, par l'imposition du discours canonique, la dynamique des processus de significations. Celle-ci se pratique dans le Talmud, aspect oral de la Torah, qui est envisagée dans sa double dimension de Mishna ou loi orale codifiée et de Gemara ou débat au sujet de la loi orale. La Torah, comme aspect élargi du texte écrit, de la loi orale et du débat au sujet de celle-ci, s'enracine dans une origine qui ouvre sur une production de significations s'accumulant et qui sont, elles aussi, Torah, enseignement sacré. Un écrivain contemporain, Edmond Jabès, le rappelle dans Le livre des ressemblances ${ }^{10}$ en mettant en place un CE NE SERA JAMAIS ÇA catalyseur d'une production de significations sans fin à partir de la lettre.

Dans ce cadre, l'origine ne peut être répétée car rien ne bloque l'ouverture individuelle vers un futur. Il n'y a ni parole privilégiée, ni institution qui affirme qu'elle peut arrêter cette production. Pour les catholiques, par contre, le Nouveau Testament est exemplaire. Il représente la lecture de l'Ancien Testament. Il en révèle le secret. La lettre de l'AT est structurée dans la cohérence du NT, allégorie du premier, c'est-à-dire commentaire canonique définitif sur le premier. L'esprit du NT dit la lettre de l'AT comme lecture accomplissant la lettre de la loi dans l'arrêt de l'excès de sens. Le NT répète en un sens l'AT en l'éclairant. L'origine est retrouvée, l'original est là comme référent ultime, comme exemple d'une parole privilégiée dont l'authenticité peut être dite. Le règne de la mimésis platonicienne s'intègre dans le fonctionnement de l'herméneutique occidentale par la révélation.

9 Voir l'édition de C. Swan, New York, AMS Press, 1970.

10 Edmond Jabès, Le livre des ressemblances, Paris, Gallimard, 1976. 


\section{La mort exquise}

Cette croyance en la répétition, en le retour à l'origine, en la possibilité de dire le référent ultime est rendue burlesque dans les diverses nouvelles de La mort exquise ${ }^{11}$ de Claude Mathieu qui renvoie à l'obsession du texte sacré et de la parole privilégiée:

Ils [les dîners] deviennent si ennuyeux qu'on avait l'impression

de répéter chaque semaine la dernière cène. Il y a eu jadis une

dernière cène et ce fut assez. ("Les dîners chez Rachel ", p. 25)

Et que se passa-t-il lors de cette dernière cène? L'équation entre le corps et le pain, le sang et le vin. L'esprit est la lettre dans la communion, le consensus gardé par l'institution. Il est possible d'avoir accès à la lettre, à l'origine et à l'authenticité dans la fusion. C'est là où les nouvelles de La mort exquise fictionnalisent toute cette croyance en la possibilité de retrouver la lettre, de la dire en tant que pleine.

Dans "Autobiographie", une des nouvelles de ce recueil, des chercheurs allemands et européens mettent en fiche tous les textes latins. La lettre et le rapport à l'autre obsèdent dans une quête d'un authentique à totaliser. Ils occupent un groupe d'intellectuels qui s'enferment dans la nouvelle caverne de Platon, "des salles souterraines en béton armé " 12 .

Des initiés garderaient à jamais dans ces abîmes les secrets de la vraie vie, pendant que des humains, de générations en générations, de siècles en siècles, de millénaires en millénaires, du singe au robot, auraient sur la surface de la terre, l'illusion de vivre. ("Autobiographie", p. 79)

Face à cet exemple, que fait l'auteur d' Autobiographie", dans un rapport à l'autre, la France comme partie de la latinité, de fascination et non d'exploration identitaire où se jouerait dynamiquement son américanité? Un thésaurus de La comédie bumaine de Balzac. De l'âge de 35 ans à 65 ans, il réduit son activité au 3 par 5 de la fiche puis au ronéotypé du dictionnaire qui sera envoyé à tous ceux dont le nom figure dans l'annuaire téléphonique et commence par la même initiale que lui. Face à ce monument, notre autobiographe espère atteindre une identité qui passe par la copie dans le morcelé. Il accumule la lettre tandis que le pro-

11 Claude Mathieu, La mort exquise, Montréal, CLF, 1965.

12 "Autobiographie", p. 74. 
cessus de lecture, non seulement comme production dynamique de significations mais aussi comme construction canonique réglée, lui échappe toujours. Par ce geste de collectionneur individuel, il se veut timidement, participant d'un fonctionnement institutionnel qu'il calque. Mais n'ayant pas le pouvoir institutionnel de lier les unités entre elles par la maîtrise d'une exégèse canonique allégorique réglée, sa démarche tombe dans l'insignifiant le plus complet. Il n'ouvre pas, comme certaines autobiographies, sur le roman d'apprentissage où l'homme se fait en se faisant, ce qui se produit dans Mémoires d'une jeune fille rangée de Simone de Beauvoir.

Et surtout, les lettres et les mots qu'il dessine, ici pour vous, ne cessent de le distraire du lien logique qu'il veut mettre entre eux, puisqu'il vous faut encore cela pour que vous compreniez. On ne saurait imaginer l'effort qu'il fait, qui le tue, pour lier les mots. ("Autobiographie", p. 88)

On peut discerner une parodie de celui qui, comme certains structuralistes orthodoxes, découpent les textes en unités et cherchent la vérité de celui-ci dans des structures plus ou moins profondes, aboutissement d'un métalangage menant à un méta-sens. Comme il envoie le texte aux inconnus dont le nom commence par la même initiale que le sien (mais on ne saura jamais son nom), la croyance à l'origine, à l'authentique, à l'accumulation d'archives et à la possibilité de dire cette origine retombe dans l'arbitraire le plus grotesque. Celui-ci est d'ailleurs évoqué dans "Le pèlerin de Bithynie" où la déesse manifeste des augures toujours favorables à l'éternel retour et au répétitif.

Il faut savoir, de plus, que notre autobiographe ne peut dire JE et qu'il ne parle de lui qu'à la troisième personne:

Avec joie, il est presque devenu fiche; aussi ne peut-il plus dire je; comme parlant d'un autre ou d'un objet, il se sert désormais de il; il se sent d'ailleurs de cette façon moins isolé, car le il implique aussi un narrateur; le je au contraire le montrerait à lui même seul et sans défense. ("Autobiographie", p. 84)

Le IL est l'aspiration à l'autorité manquante. Celle-ci passe par le texte latin, par le texte français, par le texte européen jusqu'à la communauté institutionnelle d'exégèses dualistes. Il en est marqué dans un dédoublement narrateur/moi inquiétant. Il y a quelqu'un, quelque part, qui détiendrait la vérité, une authenticité originelle, une signification essentielle, tandis que le IL qui met 
en fiche se sent comme un lecteur abandonné face au texte et à la lettre. L'aspiration au répétitif comme position fondamentale, comme connu non médiatisé dans la présence fantasmée de lecteur/auteur conjoints, s'enracine dans l'appel des idées platoniciennes ou dans l'idéal catholique de faire UN avec Dieu occupant ces deux positions. Celles-ci, jointes en un lecteur/auteur, sont essentielles chez les catholiques car la lecture de l'Ancien Testament issu de Dieu est accomplie par Jésus fils de Dieu et, en quelque sorte, par Dieu lui-même en trois personnes.

Dieu, producteur de la lettre, est aussi lecteur de celle-ci par le Nouveau Testament qui en donne l'esprit. Le jeu sur le rapport contextuel, sur le comparant/comparé, dans l'idéal catholique, forme totalité qui prend son sens, puisque Jésus n'a rien écrit mais a commenté l'AT, dans l'Église qui donne le sens de ce sens. Celui-ci doit être répété canoniquement. Le "ce ne sera jamais ça" des lectures sur lectures, dans un contexte toujours changeant et dans une optique déconstructionniste déja suggérée dans "Autobiographie", se réduit, pour le narrateur, à la vérité détenue par les spécialistes. Les autres, idéalement, n'ont d'autres activités que le mutisme de la contemplation dans la fusion avec l'origine retrouvée.

\section{Confettis}

Mignon petit chien, ne m'emporte pas, je serai gentil. Par être gentil, il entendait ne plus jamais jouer du violon, c'est-à-dire ne plus se masturber.

Freud, Totem et tabou, p. 148

Le JE, dans la nouvelle "Le voyeur" du recueil Confettis ${ }^{13}$ de Marcel Godin, n'apparaît qu'à la dernière ligne, sans nom, comme chez Claude Mathieu, mais avec la qualification que lui attribue le titre de la nouvelle, "Le voyeur". Tout au long est décrit une salle romantico-sentimentale où interagissent Germain et Viviane qui se livrent à leurs "petits exercices". On continue en affirmant que "Germain écarte légèrement les genoux... puis qu'il ose le pren-

13 Marcel Godin, Confettis, Montréal, Hurtubise HMH, 1980. 
dre dans sa main droite... qu'il le roule délicatement "14. À la fin nous apprenons, contrairement à "nos attentes", que le couple joue une partition musicale. L'un se consacre au violon, l'autre à la flute. Ce couple est le miroir de la polysémie s'emparant du jeu propre/figuré, lié aux dérivations métaphoriques et aux litotes qui ont pour rôle de nous faire lire ce qui ne doit pas être écrit.

Là, se construit une vie, celle du "voyeur" qui dit, à la dernière ligne: "J'éteins la lampe de Franz Schubert"15. Il ne peut raconter sa vie dans le cadre canonique reposant sur le positivisme du référent et des "faits" mais il s'assume comme JE surdéterminant le IL. De ce fait, est détruite la pseudo-objectivité de la narration à la troisième personne, analogue fictionnel de la postulation au métalangage comme IL non engagé et cautionné institutionnellement. Ce IL, celui du "voyeur", est un JE caché et flou assumant une épistémologie qui n'est plus celle de l'accord mimétique mais du clivage où les multiples hétérogènes sont réunis par le retour de fantasmes obsédants mais impossibles à symboliser complètement. Dans ce cas, les marques formelles autobiographiques font défaut. Une autre épistémologie ouvre sur d'autres formes au travers desquelles le genre ne se reconnaît pas immédiatement. La surprise est là comme manifestation d'une crise qui demande des lectures autres et innovatrices. Elles situent ce texte dans un paradigme générique différent maintenant toutefois un lien avec la tradition. En effet, ce texte, comme celui de Claude Mathieu, fait lire une certaine crise, celle issue de deux épistémologies en concurrence, comme disait T. S. Kuhn ${ }^{16}$. Là surgit le pouvoir de ces nouvelles manifestant avec éclat la redéfinition du sujet dans le burlesque (Mathieu) ou l'éros humoristique (Godin). Toute autobiographie est, ici, manifestée comme impossible à écrire selon les paradigmes traditionnels car le "se connaitre" est le piège par excellence. Écrire une autobiographie révèle qu'il s'agit d'un collage d'artifices masquant la pratique canonique du genre. Mais la vérité d'une vie passe ailleurs, notamment par l'inquiétante étrangeté freudienne provenant d'une structure de tiers. S'imaginer être séduit dans l'esthétique d'un rituel repris "avec une assiduité qui ne ment pas depuis des années" 17 est la

14 "Le voyeur", p. 65.

15 Ibid., p. 67.

16 T. S. Kuhn, La structure des réolutions scientifiques, Paris, Flammarion, 1972.

17 "Le voyeur", p. 65. 
24

seule autobiographie possible. N'est ce pas là l'autobiographie de tout lecteur face au texte qu'il dévore jusqu'à la dernière ligne?

Ici, l'institution monologique de la vérité monopolisée ne gouverne pas; le "ce n'est jamais ça" d'une production de significations dynamiques où lecture/écriture ne sont pas séparées s'impose. Il n'y a plus ceux qui écrivent et ceux qui disent ce qu'il faut lire et comprendre en une herméneutique construisant le sens d'une vie fondée sur la mimésis, mais une lecture/écriture où le lecteur invente les significations dans le polysémique. Ce lecteur saisit, contrairement au narrateur d' Autobiographie", que le retour à l'origine est impossible et qu'il n'y a que des différences dans des contextes changeants. Ceci rappelle Gilles Deleuze :

[...] deux lectures du monde, dans la mesure où l'une nous convie à penser la différence à partir d'une similitude ou d'une idée préalable, tandis que l'autre nous invite au contraire à penser la similitude ou même l'idée comme le produit d'une disparité de fond. La première définit exactement le monde des copies ou des représentations, elle pose le monde comme icône. La deuxième, contre la première, définit le monde des simulacres. Elle pose le monde lui-même comme fantasmes. ${ }^{18}$

On voir alors où se situe l'autobiographie dans le cadre de la dichotomie langages publics/langages spécialisés. Ces derniers étaient autrefois contrôlés par les clercs renvoyant à un extérieur comme secret possible à dire par l'institution. De nos jours, ces langages sont monopolisés par diverses institutions en dialogues dans des démocraties laïcisées jouant de l'accumulation des informations et de la disparité des sources de pouvoir ${ }^{19}$. Dans ce contexte, l'autobiographie construit ce lieu où l'identité est un rapport à réviser constamment face aux concerts bureaucratisés produisant vingt-quatre heures sur vingt-quatre, des discours normés. Les nouveaux intellectuels, quelque peu rassasiés des orthodoxies métalinguistiques et des paradigmes expliquant tout, tentent de voir où leurs discours pourraient rencontrer une fictionnalité omniprésente qui échapperait cependant, par quelque lieu, aux machines à discours, qu'il s'agisse du devenir historique inéluctable ou des verrouillages métalinguistiques.

18 Gilles Deleuze, Logique du sens, Paris, Minuit, 1975, p. 302.

19 Patrick Imbert, L'objectivité de la presse, Montréal, Hurtubise HMH, 1989. 


\section{Conclusion}

Le travail du critique [...] se propose franchement comme une lecture qui n'est pas une reproduction des ouvres mais plutôt une réécriture, une construction qui dérive d'elles comme une citation indice.

René Payant, Parachute, no ${ }^{\circ} 18$, printemps 1980, p. 31

Ces autobiographies mènent donc à se demander comment ne pas transformer le contingent en nécessité et parvenir à confronter des discours qui sont croyances affirmées correspondre à la réalité du monde (de la théologie aux lois du marché) ou à la vérité du texte. Le métalangage générique ou autre est donc déplacé dans cette confrontation à cette fiction autobiographique bouleversant la mimésis. Il devient simple discours autre manié par des spécialistes. Le voyeur, qui est aussi le lecteur depuis l'incipit jusqu'à la phrase finale, se laisse emporter dans le polysémique et insère, entre les lettres, les frissons de son épiderme et les productions de ses fantasmes. Point de savoir qui ne passe dans une unité corps / esprit et qui ne permette de parvenir à se situer dans l'équilibre temporaire du mouvement fort éloigné du dualisme mimétique connu. 